\title{
Two Methods for Estimation of Amplifier Imbalances in Multi-Amplifier Transmission Structures
}

\author{
Paulo MONTEZUMA ${ }^{1,2,3}$, Rui DINIS ${ }^{1,2}$, Sara RIBEIRO ${ }^{1}$, Marko BEKO $^{3}$ \\ ${ }^{1}$ Dept. de Engenharia Electrotecnica Faculdade de Ciências e Tecnologia (DEE-FCT), Universidade Nova de Lisboa, \\ Quinta da Torre, 2829-516 Caparica, Portugal \\ 2 Instituto de Telecomunicações, Instituto Superior Técnico, Av. Rovisco Pais 1, 1049-001 Lisboa, Portugal \\ ${ }^{3}$ Centre of Technology and Systems (UNINOVA-CTS), Universidade Nova de Lisboa, Quinta da Torre, 2829-516 Caparica, \\ Portugal
}

pmc@duninova.pt,rdinis@fct.unl.pt

Submitted May 25, 2016 / Accepted October 10, 2016

\begin{abstract}
Energy efficient power amplification of multilevel constellations can be achieved by an amplification structure based on the constellation's decomposition as a sum of polar components, such as M BPSK (Bi-Phase Shift Keying), that are separately amplified. By doing this one can define highly efficient transmitters based on multiple amplifiers. However, amplifiers' imbalances might lead to substantial constellation distortion since phase and gain imbalances cause rotations and translations of the symbols associated to each amplification branch that are combined to generate the resulting constellation. Therefore, it becomes crucial the knowledge of the amplifiers' imbalances to overcome this problem at the receiver side. For that we propose and evaluate efficient two new methods for estimating amplifier imbalances. Simulation results demonstrate that the good performance attainable by the proposed estimate algorithms can be assured without significant increase in system and computational complexity.
\end{abstract}

\section{Keywords}

Multilevel constellations, multi-amplifier transmitters, estimation

\section{Introduction}

Multilevel constellations allow a high number of information bits per dimension which, therefore, leads to higher spectral efficiency. However, this is achieved with increased power requirements since the power efficiency grows exponentially with the spectral efficiency. Moreover, the signals associated to large and dense constellations, which are the ones with lower power requirements for a given spectral efficiency [1], [2], have higher envelope fluctuations and PAPR (Peak-to-Average Power Ratio). This means additional power amplification constraints, since quasi-linear amplifiers with high back-off and much lower amplification efficiency than nonlinear amplifiers (such as class D, E or F amplifiers) are required [3].
To overcome these difficulties, a promising technique was recently proposed in [4]. The basic idea behind this technique is to decompose the signal associated to a given multilevel constellation as a sum of several uncorrelated polar components [4], [5]. Each polar component can be used to modulate a BPSK signal with reduced envelope fluctuations or even a constant envelope (e.g., using the serial MSK (Minimum Shift Keying) format [6], [7]). Therefore, each polar component can be amplified by a different low-cost and highly-efficient, nonlinear power amplifier, and then combined to generate a symbol belonging to an amplified version of the original signal (without distortion), which leads to a very efficient transmitter. In such a structure, the amplifiers of the several branches should be perfectly matched to avoid phase imbalances, otherwise the different phase rotations and translations of the polar components associated to each amplification branch will cause cumulative distortion effects of the resulting symbol after the combiner. Thus, imbalances in the phase and/or gains of the different amplifiers might lead to substantial constellation distortion, and, consequently, significant performance degradation, unless the receiver is modified to take into account the actual constellations distortion [8]. Since amplifiers' operation conditions may vary with time, imbalances may occur even when amplifiers were perfectly matched in setup phase. To compensate this effect several techniques have been proposed to control amplifiers offset [9-12], but despite the offset compensation residual imbalances may occur. Moreover, most part of these compensation techniques consider two amplifiers and their extension to a higher number of amplifiers can relax the tolerance of the compensated offset, which may affect the system performance when high order constellations are employed. Therefore, it would be useful to have an algorithm to estimate the amplifiers' imbalances and constellation distortion at the receiver side.

At this point it becomes obvious that the receiver should know the transmitter's amplifiers coefficients $g_{i}$ and the amplifiers' imbalances. Obviously, it is also important to evalu- 
ate the impact of those imbalances but the scope of this work is restricted to the imbalance estimation. For that purpose pilots sent by the transmitter can be used at receiver's side for estimation of amplifiers imbalances. The drawback of such approach lies on the high number of pilots needed that may compromise spectral efficiency. To minimize the impact on spectral efficiency the data estimations can used together with the pilots. Therefore, in this paper we propose and evaluate decision-directed (DD) and data-aided (DA) techniques for estimating the amplifiers' imbalances and constellation distortion at the receiver side.

This paper is organized as follows: signal and system characterizations are done in Sec. 2. The problem formulation and the imbalance estimation algorithms are presented in Sec. 3. Performance results of both algorithms are presented and compared in Sec. 4. Section 5 resumes this paper.

\section{Signal Characterization}

Multilevel constellations can be decomposed in polar components. To do this let us consider a constellation $\mathcal{S}=$ $\left\{s_{0}, s_{1}, \ldots, s_{(M-1)}\right\}$, with $M$ points (i.e., $\# \mathcal{S}=M$ ), where $s_{n} \in \mathbb{C}$. To each constellation point $s_{n}$ we associate a set of $\mu=\log _{2}(M)$ bits $\mathcal{B}=\left\{b_{n}^{0}, b_{n}^{1}, \ldots, b_{n}^{(\mu-1)}\right\}$. It is shown in [5] that

$$
s_{n}=\sum_{m=0}^{M-1} g_{m} \prod_{i=0}^{\mu-1}\left(b_{n}^{(i)}\right)^{\gamma_{(i, m)}}
$$

where $\left(\begin{array}{lllll}\gamma_{\mu-1, i} & \gamma_{\mu-2, i} & \ldots & \gamma_{2, i} & \gamma_{0, i}\end{array}\right)$ denotes the binary representation of $i$.

In matrix format we have $\mathbf{s}=\mathbf{W g}$, where $\mathbf{W}$ is an appropriate Hadamard matrix, $\mathbf{s}=\left[\begin{array}{llll}s_{0} & s_{1} & \ldots & s_{M-1}\end{array}\right]^{T}$ denotes the ordered constellation and $\mathbf{g}=\left[\begin{array}{llll}g_{0} & g_{1} & \ldots & g_{M-1}\end{array}\right]^{T}$ denotes the ordered set of amplifiers' gains and [.. $]^{T}$ denotes the transpose. Similarly, $\mathbf{g}=\mathbf{W}^{-1} \mathbf{s}$, i.e., there is a straightforward relation between the constellation points $\mathbf{S}$ and the amplifiers' gains $\mathbf{g}$. The transmitter structure of [4] takes advantage of this decomposition in polar components, which are used to modulate several BPSK signals with reduced envelope fluctuations and the resulting signals are amplified by different low-cost and highly-efficient, nonlinear power amplifiers.

It can be shown that a multilevel constellations may be expressed as a sum of BPSK components and the constellation symbols can be expressed as a function of the corresponding bits. Consider a constellation $\mathcal{S}=\left\{s_{0}, s_{1}, \ldots\right.$, $\left.s_{(M-1)}\right\}$, with $M$ points (i.e., \#S $=M$ ), where $s_{n} \in \mathbb{C}$. To each constellation point $s_{n}$ we associate a set of $\mu=\log _{2}(M)$ bits $\mathcal{B}=\left\{b_{n}^{0}, b_{n}^{1}, \ldots, b_{n}^{(\mu-1)}\right\}$. Actually, the bits are in polar format, i.e., $b_{n}^{(i)}= \pm 1=2 \beta_{n}^{(i)}-1, \beta_{n}^{(i)}=0$ or 1 . The set of $\mu$ bits can be decomposed in $M=2^{\mu}$ different subsets $\mathcal{B}_{m}$, $m=0,1, \ldots, M-1$. Since we have $M$ constellation points in $\mathcal{S}$ and $M$ different subsets of $\mathcal{B}, \mathcal{B}_{0}, \mathcal{B}_{1}, \ldots, \mathcal{B}_{M-1}$, we can write

\begin{tabular}{|c|c|c|c|}
\hline $\mathrm{m}$ & $\gamma_{(1, m)}, \gamma_{(0, m)}$ & $\mathcal{B}_{m}$ & $\prod_{i=0}^{\mu-1} b_{n}^{(i)^{\gamma^{(i, m)}}}$ \\
\hline 0 & 00 & $\emptyset$ & 1 \\
1 & 01 & $\left\{b_{n}^{(0)}\right\}$ & $b_{n}^{(0)}$ \\
2 & 10 & $\left\{b_{n}^{(1)}\right\}$ & $b_{n}^{(1)}$ \\
3 & 11 & $\left\{b_{n}^{(0)}, b_{n}^{(1)}\right\}$ & $b_{n}^{(0)} b_{n}^{(1)}$ \\
\hline
\end{tabular}

Tab. 1. subsets $\mathcal{B}$ and constellation symbols relation.

\begin{tabular}{|c|c|c|c|}
\hline $\mathrm{m}$ & $s_{m}$ & $\beta_{n}^{(1)} \beta_{n}^{(0)}$ & $b_{n}^{(1)} b_{n}^{(0)}$ \\
\hline 0 & -3 & 00 & $-1-1$ \\
1 & -1 & 01 & $-1+1$ \\
2 & 1 & 11 & $+1+1$ \\
3 & 3 & 10 & $+1-1$ \\
\hline
\end{tabular}

Tab. 2. Symbol and bit mapping.

$$
s_{n}=\sum_{m=0}^{M-1} g_{m} \prod_{b_{n}^{(i)} \in \mathcal{B}_{m}} b_{n}^{(i)}, n=0,1, \ldots, M-1
$$

which corresponds to a system of $M$ equations (one for each $s_{n}$ and $M$ unknown variables $g_{m}$ ). Without loss of generality, we can associate $m$ to its corresponding binary representation with $\mu$ bits, i.e., $m=\left(\gamma_{(\mu-1, m)}, \gamma_{(\mu-2, m)}, \ldots, \gamma_{(1, m)}, \gamma_{(0, m)}\right)$ and define $\mathcal{B}_{m}$ as the set of bits where the bit $b_{n}^{(i)}$ is included if and only $\gamma_{(i, m)}$ is 1 . For example, considering the 4 PAM constellation $(M=4)$ and $\mathcal{S}=\{-3,-1,1,3\}$, the corresponding $\mu=2$ bits are $b_{n}^{0}$ and $b_{n}^{1}$, i.e., $\mathcal{B}=\left\{b_{n}^{(0)}, b_{n}^{(1)}\right\}$ and $\mathcal{B}$ has the following 4 subsets $\phi,\left\{b_{n}^{(0)}\right\},\left\{b_{n}^{(1)}\right\}$ and $\mathcal{B}=\left\{b_{n}^{0}, b_{n}^{1}\right\}$ of Tab. 1. Therefore, we may write

$$
\begin{aligned}
& s_{0}=g_{0}+g_{1} b_{0}^{(0)}+g_{2} b_{0}^{(1)}+g_{3} b_{0}^{(0)} b_{0}^{(1)}, \\
& s_{1}=g_{0}+g_{1} b_{1}^{(1)}+g_{2} b_{1}^{(1)}+g_{3} b_{1}^{(0)} b_{1}^{(1)}, \\
& s_{2}=g_{0}+g_{1} b_{2}^{(1)}+g_{2} b_{2}^{(1)}+g_{3} b_{2}^{(0)} b_{2}^{(1)}, \\
& s_{3}=g_{0}+g_{1} b_{3}^{(1)}+g_{2} b_{3}^{(1)}+g_{3} b_{3}^{(0)} b_{3}^{(1)}
\end{aligned}
$$

which in matrix format, $\mathbf{s}=\mathbf{W g}$ results

$$
\left[\begin{array}{l}
s_{0} \\
s_{1} \\
s_{2} \\
s_{3}
\end{array}\right]=\left[\begin{array}{cccc}
1 & b_{0}^{(0)} & b_{0}^{(1)} & b_{0}^{(0)} b_{0}^{(1)} \\
1 & b_{1}^{(0)} & b_{1}^{(1)} & b_{1}^{(0)} b_{1}^{(1)} \\
1 & b_{2}^{(0)} & b_{2}^{(1)} & b_{2}^{(0)} b_{2}^{(1)} \\
1 & b_{3}^{(0)} & b_{3}^{(1)} & b_{3}^{(0)} b_{3}^{(1)}
\end{array}\right]\left[\begin{array}{l}
g_{0} \\
g_{1} \\
g_{2} \\
g_{3}
\end{array}\right] .
$$

Let us consider now the mapping in Tab. 2. In this case we have

$$
\mathbf{W}=\left[\begin{array}{rrrr}
1 & -1 & -1 & 1 \\
1 & 1 & -1 & -1 \\
1 & 1 & 1 & 1 \\
1 & -1 & 1 & -1
\end{array}\right]
$$

Since $\mathbf{g}=\mathbf{W}^{-1} s=\frac{1}{4} \mathbf{W}^{T} \mathbf{S}=\frac{1}{4} \Leftrightarrow \mathbf{g}=\frac{1}{4}$ we may write

$$
\mathbf{g}=\left[\begin{array}{rrrr}
1 & 1 & 1 & 1 \\
-1 & 1 & 1 & -1 \\
-1 & -1 & 1 & 1 \\
1 & -1 & 1 & -1
\end{array}\right]\left[\begin{array}{r}
-3 \\
-1 \\
1 \\
3
\end{array}\right]
$$

Thus, $g_{0}=0, g_{1}=0, g_{2}=2, g_{3}=-1$, which means $s_{n}=2 b_{n}^{(1)}-b_{n}^{(1)} b_{n}^{(2)}$. Therefore, we have $\mathbf{g}=\mathbf{W}^{-1} s$. 


\section{Estimation of Amplifiers' Imbal- ances and Distorted Constellation}

Once that

$$
\prod_{b_{n}^{(i)} \in \mathcal{B}_{m}} b_{n}^{(i)}=\prod_{i=0}^{\mu-1} b_{n}^{(i)^{\gamma(i, m)}}=b_{n}^{m(e q)}= \pm 1
$$

each of the $M$ components can be regarded as a BPSK signal of the type

$$
x_{m}(t)=\left|g_{m}\right| \cos \left(2 \pi f_{c} t+\varphi^{m(e q)}+\arg \left(g_{m}\right)\right)
$$

with

$$
\varphi^{m(e q)}=\left\{\begin{array}{c}
0, b_{n}^{m(e q)}=1 \\
\pi, b_{n}^{m(e q)}=-1
\end{array}\right\}
$$

which leads to a transmitter structure with $M$ amplifiers characterized by gains $\left|g_{m}\right|$ and phases $\arg \left(g_{m}\right)$. Due to hardware impairments the amplifiers' complex gains $g_{m}=$ $g_{m}^{\text {nom }}+\varepsilon_{m}^{g}, m=0,1, \ldots, M-1$, with $\varepsilon_{m}^{g}$ associated to gain and phase errors and $g_{m}^{\text {nom }}$ the nominal gain of the amplifier. From these gains we will have the constellations, because $\mathbf{s}=\mathbf{W g}_{m}$ with

$$
\mathbf{s}=\left[\begin{array}{c}
s_{0} \\
s_{1} \\
\ldots \\
s_{M-1}
\end{array}\right] \text { and } \mathbf{g}=\left[\begin{array}{c}
g_{0} \\
g_{1} \\
\ldots \\
g_{M-1}
\end{array}\right]
$$

As referred before, there is a linear and invertible relation between constellation points $\mathbf{s}$ and amplifiers' gains $\mathbf{g}$. So, by solving one estimation problem we are also solving the other. Therefore, our goal is to estimate the actual constellation $\mathcal{S}=\left\{s_{0}, s_{1}, \ldots, s_{M-1}\right\}$ and, inherently, the actual gains, from the received symbols $y_{m}=x_{m}+\varepsilon_{m}, m=0,1, \ldots, N-1$ (the index $m$ is the symbol number, not the constellation point before) and $x_{m} \in \mathcal{S}$. For that purpose we can adopt one of the two methods characterized in the following subsections and we assume Gaussian noise with zero mean, i.e., $\varepsilon_{n} \sim N\left(0, \sigma^{2}\right)$.

\subsection{Data Aided Estimation}

Let us assume that the transmitted symbols are known by the receivers (i.e., the $s_{n}, n=0,1, \ldots, N-1$, correspond to an appropriate training sequence). In that case the optimum estimates of $s_{n}$ are

$$
\hat{s}_{n}=\frac{1}{N_{n}} \sum_{m, x_{m}=s_{n}} y_{m}
$$

where $N_{n}=\#\left\{x_{m}: x_{m}=s_{n}\right\}$, i.e., is the number of symbols where $x_{m}=s_{n}$ and $y_{m}$ represents the received symbol. For $N \gg M$ we have $N_{m} \simeq \frac{N}{M}$ (if we assume equally probable constellation symbols) and we may give the estimation as

$$
\hat{s}_{n}=\hat{\alpha}_{n} s_{n}+\hat{\varepsilon}_{n} .
$$

Since the estimation is unbiased, we have $\alpha_{n}=1$ and $\hat{s}_{n} \sim \mathcal{C N}\left(s_{n}, \sigma_{\varepsilon}^{2}\right)$, where $\sigma_{\varepsilon}^{2}=\mathrm{E}\left[\left|\hat{\varepsilon_{n}^{2}}\right|\right]=\mathrm{E}\left[\left|\hat{s_{n}}-s_{n}\right|^{2}\right]=$ $\frac{\sigma^{2}}{N_{m}} \simeq \frac{N}{M} \sigma^{2}$. Thus, from $\left\{\hat{s}_{n}, n=0,1, \ldots, M-1\right\}$ we can obtain $\left\{\hat{g}_{n}, n=0,1, \ldots, M-1\right\}$, since $\hat{\mathbf{g}}=\mathbf{W}^{-1} \hat{\mathbf{s}}$ where $\hat{g}=\alpha_{n}^{g} g_{n}+\varepsilon_{n}^{g}$.

\subsection{Decision-Directed Estimation}

The main problem of DA estimate is the need of $N \gg M$ training symbols, which may have impact on power and spectral efficiencies due to the higher number of pilots needed. When a reasonable estimate of the adopted constellation $\tilde{\mathcal{S}}=\left\{s_{n}, n=0,1, \ldots, M-1\right\}$ is available (e.g., for slowvarying scenarios we can obtain $\tilde{S}$ from a training sequence and use it for several data blocks) and the SNR (signal to noise ratio) is not too low, it is possible to use a DD estimation of $\tilde{\mathcal{S}}$. The basic procedure is as follows: A set of $N$ data symbols $x_{m} \in \mathcal{S}, m=0,1, \ldots, N-1$ is transmitted, being the symbols unknown by the receiver. The corresponding received symbols are $y_{m}=x_{m}+\varepsilon_{m}$. From these symbols we obtain the estimates of the transmitted symbols $\hat{x}_{m}=s_{n}$ that minimizes $\left|y_{n}-s_{n}\right|^{2}$ with $s_{n} \in \hat{\mathcal{S}}$ (for more details see Appendix). From $x_{m}$ we have

$$
\hat{s}_{n} \simeq \frac{1}{N_{n}} \sum_{m, \hat{x}_{m}=s_{n}} y_{n} \simeq \frac{M}{N} \sum_{m, \hat{x}_{m}=s_{n}} y_{n} .
$$

Once again, we have $\hat{\mathbf{g}}=\mathbf{W}^{-1} \hat{\mathbf{s}}$ and $\hat{\mathbf{s}}=\alpha_{n}^{s} s_{n}+\varepsilon_{n}^{s}$ and $\hat{\mathbf{g}}=\alpha_{n}^{g} g_{n}+\varepsilon_{n}^{g}$.

\section{Performance Results}

We consider the use of the multi-amplifier structure of [4] for efficient amplification of the signals associated to multilevel constellations and a set of performance results concerning the estimation of the distorted constellation due to amplifiers' imbalances is presented. For the sake of simplicity, we assume an ideal AWGN channel (Additive White Gaussian Noise) and mainly 16QAM constellations. All the constellations are normalized to have unitary energy and it is assumed that the amplifiers' gains have uncorrelated, zeromean Gaussian errors with variance $\sigma_{g}^{2}$.

First we start with a series of results regarding the effect of $N$ and $\sigma_{g}^{2}$ in regular 16QAM constellations, i.e, a transmitter with 4 amplification branches in parallel. For a comparative analysis of the estimation error $\sigma_{\varepsilon_{S}}^{2}$ with the type and constellation sizes, Voronoi constellations and other constellation sizes are also included in the last results presented here. It should be mentioned that the results presented here can easily be extended to other channels, provided that appropriate equalizers are employed as well as other constellation types. Since our estimator is unbiased, in the DA estimation 


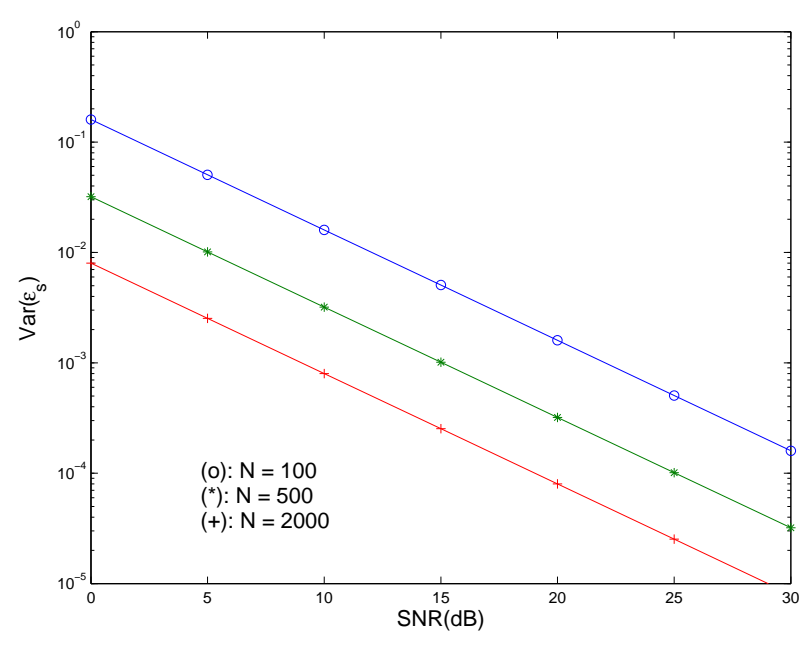

Fig. 1. Estimation error for the data-aided estimator with different values of $N$ (markers denote simulated values).

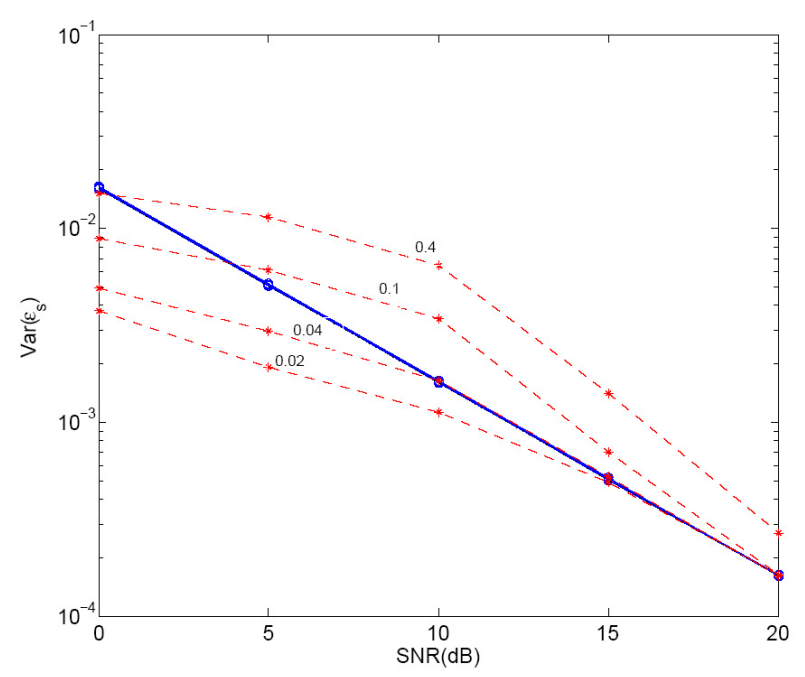

Fig. 2. Estimation error for the data-aided estimator (solid line) and the decision-directed (dashed-line) different values of $\sigma_{g}^{2}$.

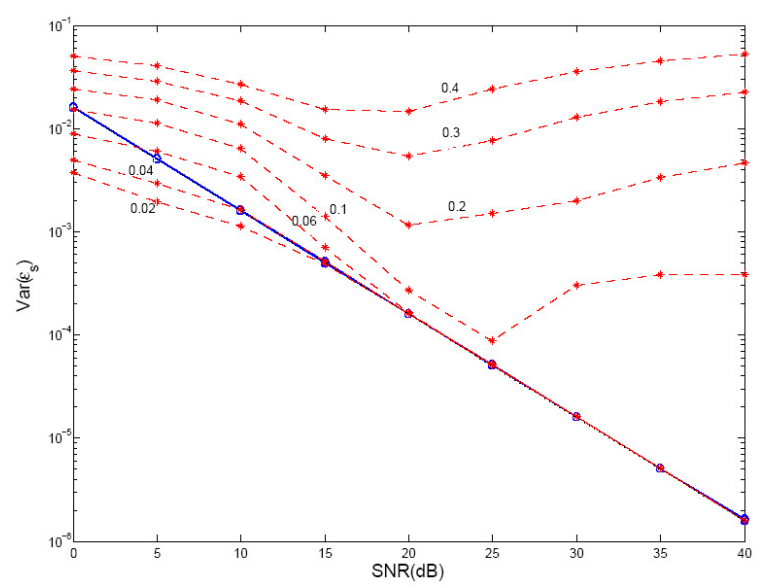

Fig. 3. Behavior of estimation error for 16 QAM constellation with different values of $\sigma_{g}$.

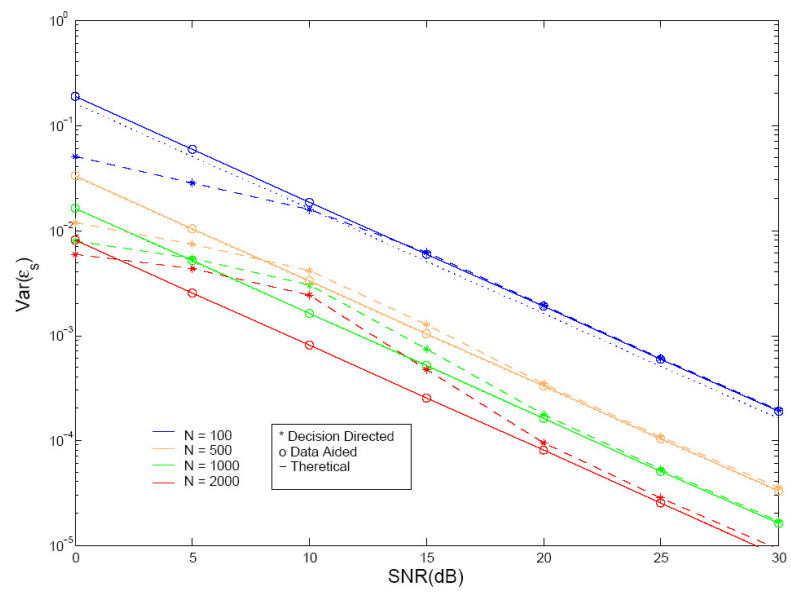

Fig. 4. Estimation error for 16 QAM constellation and different values of $N$.

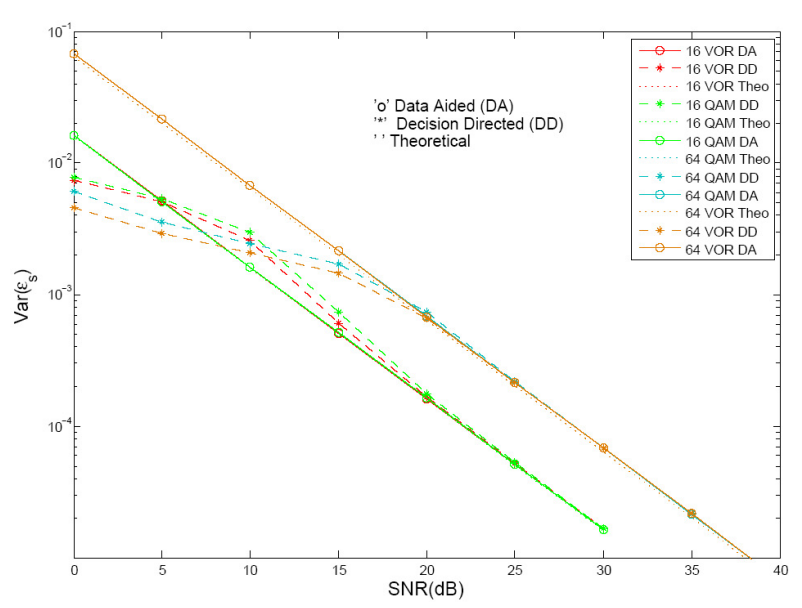

Fig. 5. Estimation error comparison between different constellations with $N=1000$.

case (and approximately unbiased in the DD case) it will be enough to study the variance of the estimators to evaluate their performance. We only present results for the distorted constellation estimation error $\sigma_{\varepsilon_{S}}^{2}$.

Figure 1 shows the variance of the estimation error for different training sequence durations. It can be seen that there is a perfect matching between theoretical and estimated results and, as expected, the performance improves with the duration of the training sequence and the SNR. Figure 2 compares DA and DD estimators with $N=1000$. Clearly, the DD estimator has excellent performance, provided that the errors in the amplifiers' gains are not too high (the apparent advantage of the DD estimator for low SNR is due to decision errors). As expected, larger constellations require smaller values of $\sigma_{g}^{2}$ for a good performance of the DD estimator. For the DA estimator we have similar estimation performance regardless of $\sigma_{g}^{2}$.

In Fig. 3 it is shown the impact of $\sigma_{g}$ in the estimation algorithms for a 16-QAM constellation with $N=100$. These results confirm previous behavior of DA estimation, since it has excellent performance (near to the theoretical one) regardless the value of $\sigma_{g}$. On the other hand the DD 
estimator only converges to the optimal performance when $\sigma_{g} \leq 0.06$. For the other constellations, this type of estimation only works if $\sigma_{g}$ value is approximately zero.

From the estimation error results presented in Fig. 4 (for a 16 QAM constellation with different $N$ values and $\left.\sigma_{g} \leq 0.06\right)$, it is clear that with the increase of $N$ and SNR values, $\epsilon_{S}$ variance is meaningly better. With only the growth of the SNR this fact is true as well.

Figure 5 compares estimation performances for different constellations and an unique $N(N=1000)$. Since constellations with equal number of bits have the same theoretical performance, the DA's performance line is equal for these constellations too. Comparing results obtained for Voronoi and QAM constellations it becomes clear that Voronoi constellations have worst performance than QAM for the DD case (this is due to the fact that Voronoi constellation have more components in their definition and consequently any estimation error has an higher impact on performance). The DA estimation technique is always coincident with theoretical values to all SNR and $\sigma_{g}$ values. Contrary, the DD only converges to ideal result from a specific value of SNR and when $\sigma_{g}$ is near zero. Therefore, we may conclude that the larger a constellation is, smaller is the $\sigma_{g}$ that allows the convergence of the DD estimation technique.

\section{Conclusions}

In this paper we considered the problem of imbalances in structures using multiple amplifiers for an efficient amplification of multilevel constellations. We proposed efficient methods for estimating amplifiers' gains and the corresponding distorted constellation at the receiver. Despite the good performance of both methods, it was shown that we may avoid the $N \gg M$ training symbols of DA estimation method by adopting a DD technique. It was also shown that the DD technique can estimate de transmitted symbols when is available information about the constellation. This process of estimation is less tolerant to errors and imbalances than the DA estimation. The results have shown that when less errors and deviations can be assured, the DD technique has a perfect performance and is a better choice than the other method.

\section{Acknowledgments}

This work was partially supported by Portuguese Foundation for Science and Technology under grants SFRH/ BPD/108232/2015 and Science 2008 Post-Doctoral Research grant, as well as projects UID/EEA/50008/2013 (Portuguese Telecommunications Institute) and PEst-OE/EEI/UI0066/2014 (UNINOVA), project PTDC/EEI-TEL/6308/2014-HAMLeT, GALNC EXPL/EEI-TEL/1582/2013 and Program Investigador FCT (IF/00325/2015).

\section{References}

[1] FOSCHINI, G., GITLIN, R., WEINSTEIN, S. Optimization of two dimensional signal constellations in the presence of Gaussian noise. IEEE Transactions on Communications, 1974, vol. 22, no. 1, p. 28-38. DOI: 10.1109/TCOM.1974.1092061

[2] BEKO, M., DINIS, R. Designing good multi-dimensional constellations. IEEE Wireless Communications Letters, 2012, vol. 1, no. 3. DOI: 10.1109/WCL.2012.032312.120203

[3] KEE, S., AOKI, I., HAJIMIRI, A., et al. The class-E/F family of ZVS switching amplifiers. IEEE Transactions on Microwave Theory and Techniques, 2003, vol. 51, no. 6, p. 1677-1690. DOI: 10.1109/TMTT.2003.812564

[4] ASTUCIA, V., MONTEZUMA, P., DINIS, R., et al. On the use of multiple grossly nonlinear amplifiers for higly efficient linear amplification of multilevel constellations. In Proceedings of the IEEE 78th Vehicular Technology Conference (VTC Fall). Las Vegas, NV (USA), 2013, p. 1-5. DOI: 10.1109/VTCFall.2013.6692320

[5] DINIS, R., MONTEZUMA, P., SOUTO, N., et al. Iterative frequencydomain equalization for general constellations. In Proceedings of the 33rd IEEE Sarnoff Symposium. Princeton, NJ (USA), 2010, p. 1-5. DOI: $10.1109 /$ SARNOF.2010.5469792

[6] AMOROSO, F., KIVETT, J. Simplified msk signalling technique. IEEE Transactions on Communications, 1977 , vol. 25, no. 4 DOI: 10.1109/TCOM.1977.1093835

[7] SVEDEK, T., HERCEG, M., MATIC, T. A simple signal shaper for GMSK/GFSK and MSK modulator based on sigma-delta look-up table. Radioengineering, 2009, vol. 18, no. 2. ISSN: 1805-9600

[8] MONTEZUMA, P., MARQUES, D., ASTUCIA, V., et al. Robust frequency-domain receivers for a transmission technique with directivity at the constellation level. In Proceedings of the IEEE 80th Vehicular Technology Conference (VTC Fall). Vabcouver (Canada), 2014, p. 1-7. DOI: 10.1109/VTCFall.2014.6966163

[9] SOUliOTIS, G., LAOUDIAS, C., TERZOPOUlOS, N. An offset cancelation technique for latch type sense amplifiers. Radioengineering, 2014, vol. 23, no. 4. ISSN: 1805-9600

[10] WONG, K.-L. J., YANG, C.-K. K. Offset compensation in comparators with minimum input-referred supply noise. IEEE Journal of Solid-State Circuits, 2004, vol. 39, no. 5, p. 837-840. DOI: $10.1109 /$ JSSC.2004.826317

[11] KHANGHAH, M. M., SADEGHIPOUR, K. D. A $0.5 \mathrm{~V}$ offset cancelled latch comparator in standard $0.18 \mu \mathrm{m}$ CMOS process. Analog Integrated Circuits and Signal Processing, 2014, vol. 79, no. 1, p. 161-169. DOI: $10.1007 / \mathrm{s} 10470-013-0239-\mathrm{z}$

[12] CHEN, J., LI, G., CHENG, Y. Low-power offset-cancellation switched-capacitor correlated double sampling bandgap. Electronics Letters, 2012, vol. 48, no. 14, p. 821-822. DOI: 10.1049/el.2012.0857

[13] KAY, S. M. Fundamentals of Statistical Signal Processing: Estimation Theory. Upper Saddle River, NJ (USA): Prentice-Hall, 1993.

\section{About the Authors...}

Rui DINIS received the Ph.D. degree from Instituto Superior Tecnico (IST), Technical University of Lisbon, Portugal, in 2001 and the Habilitation in Telecommunications from Faculdade de Ciencias e Tecnologia (FCT), Universidade Nova de Lisboa (UNL), in 2010. His main research activities are on modulation and transmitter design, signal processing, 
nonlinear effects on digital communications and receiver design, with emphasis on frequency-domain implementations, namely for MIMO systems and/or OFDM and SC-FDE modulations.

Paulo MONTEZUMA received his $\mathrm{PhD}$ from FCTUniversidade Nova de Lisboa. He has been actively involved in several international research projects in the broadband wireless communications area and many national projects. His main research activities are on modulation and transmitter design, coding, nonlinear effects on digital communications and receiver design, with emphasis on frequencydomain implementations, namely for single carrier and multicarrier modulations.

Sara RIBEIRO received the M.Sc degree from from Faculdade de Ciencias e Tecnologia (FCT), Universidade Nova de Lisboa (UNL), in 2015. His main research activities are on multi-antenna transmitter design, OFDM and SC-FDE modulations.

Marko BEKO was born in Belgrade, Serbia, on November 11, 1977. He received the Dipl. Eng. degree from the University of Belgrade, Belgrade, Serbia, in 2001 and the $\mathrm{PhD}$ degree in electrical and computer engineering from Instituto Superior Tecnico, Lisbon, Portugal, in 2008. Currently, he is an Associate Professor at the Universidade Lusófona de Humanidades e Tecnologias, Portugal. He is also a Researcher at the UNINOVA, Campus da FCT/UNL, Monte de Caparica, Portugal. His current research interests are in the area of signal processing for wireless communications and nonsmooth and convex optimization. He is the winner of the 2008 IBM Portugal Scientific Award.

\section{Appendix: Parameters Estimation}

The set of $N$ symbols consists in a set of $N$ samples of a random variable $x_{n}=\mu+\varepsilon_{n}, n=0,1, \ldots, N-1$ where $\mu$ is constant and $\varepsilon \sim N\left(0, \sigma^{2}\right)$. The idea behind ML (Maximum likelihood) estimation [13] is to obtain the estimates for $\mu$ and $\sigma^{2}$ that maximize

$$
p\left(\mu, \sigma^{2} \mid x_{n}\right)
$$

For this propose, we form the loglikelihood function

$$
\log \left(p\left(\mu, \sigma^{2} \mid x_{n}\right)\right)
$$

Since the logarithmic function is monotone, maximize it is equivalent to maximize (13).

$$
p\left(\mu, \sigma^{2} \mid x_{n}\right)=\frac{p\left(x_{n} \mid \mu, \sigma^{2}\right) p\left(\mu, \sigma^{2}\right)}{p\left(x_{n}\right)} .
$$

When any a priori information for $\mu$ and $\sigma^{2}$ is unavailable, we may assume uniform distributions for both parameters. In (15) the $p\left(x_{n}\right)$ can be regarded as a constant with respect to $\mu$ and $\sigma^{2}$. Therefore, maximizing (14) is formally equivalent to maximize

$$
\begin{aligned}
L\left(\mu, \sigma^{2}\right) & =\log \left(p\left(x_{n} \mid \mu, \sigma^{2}\right)\right)=\log \left(\prod_{n=0} p\left(x_{n} \mid \mu, \sigma^{2}\right)\right) \\
& =\sum_{n=0}^{N-1} \log \left(p\left(x_{n} \mid \mu, \sigma^{2}\right)\right) .
\end{aligned}
$$

Since $\varepsilon \sim \mathcal{C N}\left(0, \sigma^{2}\right)$ then $x_{n} \mid \mu, \sigma^{2} \sim \mathcal{C N}\left(\mu, \sigma^{2}\right)$ and $p\left(x_{n} \mid \mu, \sigma^{2}\right)=\frac{1}{\sqrt{2 \pi \sigma^{2}}} e^{-\frac{\left|x_{n}-\mu\right|^{2}}{2 \sigma^{2}}}$. Therefore, we may write

$$
L\left(\mu, \sigma^{2}\right)=-\sum_{n=0}^{N-1} \frac{\left(x_{n}-\mu\right)^{2}}{2 \sigma^{2}}-\frac{N}{2} \log \left(2 \pi \sigma^{2}\right) .
$$

Function $L\left(\mu, \sigma^{2}\right)$ reaches a maximum when

$$
\begin{aligned}
& \frac{\partial L}{\partial \mu}=0 \\
& \frac{\partial L}{\partial \sigma^{2}}=0
\end{aligned}
$$

which is equivalent to have

$$
\sum_{n=0}^{N-1} 2\left(x_{n}-\mu\right)=0 \Leftrightarrow \mu=\frac{1}{N} \sum_{n=0}^{N-1} x_{n},
$$

and

$$
\begin{gathered}
\frac{1}{\sigma^{3}} \sum_{n=0} N-1\left(x_{n}-\mu\right)^{2}-\frac{N}{\sigma} \\
\Rightarrow \sigma^{2}=\frac{1}{N} \sum_{n=0}^{N-1}\left(x_{n}-\mu\right)^{2} .
\end{gathered}
$$

